\title{
Structural brain imaging studies offer clues about the effects of the shared genetic etiology among neuropsychiatric disorders
}

\author{
Nevena V. Radonjić $\mathbb{D}^{1} \cdot$ Jonathan L. Hess $\mathbb{D}^{2} \cdot$ Paula Rovira $\mathbb{D}^{3,4} \cdot$ Ole Andreassen ${ }^{5}$ - Jan K. Buitelaar $\mathbb{D}^{6,7,8}$. \\ Christopher R. K. Ching ${ }^{9}$. Barbara Franke $\mathbb{D}^{7,10,11} \cdot$ Martine Hoogman $^{10} \cdot$ Neda Jahanshad $\mathbb{D}^{12} \cdot$ Carrie McDonald $^{13}$. \\ Lianne Schmaal $\mathbb{1}^{14,15}$. Sanjay M. Sisodiya ${ }^{16,17}$. Dan J. Stein $\mathbb{1}^{18}$. Odile A. van den Heuvel ${ }^{19}$. \\ Theo G. M. van Erp $\mathbb{1}^{20,21} \cdot$ Daan van Rooij ${ }^{22} \cdot$ Dick J. Veltman $^{19} \cdot$ Paul Thompson $^{23} \cdot$ Stephen V. Faraone $\mathbb{C}^{2}$
}

Received: 29 May 2020 / Revised: 7 December 2020 / Accepted: 11 December 2020 / Published online: 17 January 2021

(c) The Author(s) 2021. This article is published with open access

\begin{abstract}
Genomewide association studies have found significant genetic correlations among many neuropsychiatric disorders. In contrast, we know much less about the degree to which structural brain alterations are similar among disorders and, if so, the degree to which such similarities have a genetic etiology. From the Enhancing Neuroimaging Genetics through MetaAnalysis (ENIGMA) consortium, we acquired standardized mean differences (SMDs) in regional brain volume and cortical thickness between cases and controls. We had data on 41 brain regions for: attention-deficit/hyperactivity disorder (ADHD), autism spectrum disorder (ASD), bipolar disorder (BD), epilepsy, major depressive disorder (MDD), obsessive compulsive disorder (OCD), and schizophrenia (SCZ). These data had been derived from 24,360 patients and 37,425 controls. The SMDs were significantly correlated between SCZ and BD, OCD, MDD, and ASD. MDD was positively correlated with BD and OCD. BD was positively correlated with OCD and negatively correlated with ADHD. These pairwise correlations among disorders were correlated with the corresponding pairwise correlations among disorders derived from genomewide association studies $(r=0.494)$. Our results show substantial similarities in sMRI phenotypes among neuropsychiatric disorders and suggest that these similarities are accounted for, in part, by corresponding similarities in common genetic variant architectures.
\end{abstract}

\section{Introduction}

Neuropsychiatric disorders have substantial heritability, as shown by many studies of twins and families [1]. Genomewide association studies (GWAS) have shown that common genetic variants account for some of this heritability, and that some of this heritability is shared across neuropsychiatric disorders [2-5]. The genetic overlap across disorders may partly explain why these disorders tend to cooccur with one another in both clinical and community samples [6].

Supplementary information The online version of this article (https:// doi.org/10.1038/s41380-020-01002-z) contains supplementary material, which is available to authorized users.

Stephen V. Faraone

sfaraone@childpsychresearch.org

Extended author information available on the last page of the article
Subcortical brain volumes and cortical thickness/surface area dynamically change from early development through adulthood and old age. A study of the Enhancing Neuroimaging Genetics through Meta-Analysis (ENIGMA) Plasticity Working Group reported that changes in structural magnetic resonance imaging (sMRI) phenotypes have heritabilities ranging from $5 \%$ for pallidum to $42 \%$ for cerebellar gray matter [7]. Heritability estimates of change rates were age-related and generally higher in adults than in children, probably due to an increasing influence of genetic factors with age [7]. However, it appears that later in adulthood heritability decreases most likely due to cumulative effect of environmental influences over the lifespan [8]. ENIGMA sMRI studies of different psychiatric and neurological disorders further characterized MRI-derived phenotypes that can be used to assess heritability (reviewed in [9]).

ENIGMA has also reported significant case vs. control differences in sMRI phenotypes for: attention-deficit/ hyperactivity disorder (ADHD) [10, 11], autism spectrum disorder (ASD) [12], bipolar disorder (BD) [13, 14], 
common epilepsy syndromes [15], major depressive disorder (MDD) [16, 17], obsessive compulsive disorder (OCD) [18, 19], and schizophrenia (SCZ) [20, 21]. Here we estimate the degree of similarity in sMRI phenotypes among these disorders and evaluate whether these similarities are influenced by corresponding similarities in common genetic variant architectures.

\section{Methods}

\section{Collection of structural neuroimaging summary statistics}

Summary statistics from ENIGMA structural neuroimaging studies were collected from 12 multisite analyses published by the ENIGMA Consortium for the following neuropsychiatric disorders: ADHD [10, 11], ASD [12], BD [13, 14], epilepsy [15], MDD [16, 17], OCD [18, 19], and SCZ [20, 21]. Prior to computing the summary statistics, the regional brain volumes had been segmented with a common ENIGMA protocol using FreeSurfer software. Each site performed these segmentations on their raw data. In addition, quality control protocols provided by ENIGMA were run at each site. Details are at: http://enigma.ini.usc.edu/ protocols/imaging-protocols.

The ADHD and ASD samples comprised both youth and adults. The other samples comprised adults only. The ethnicity of the patients was not available for all participants. The "epilepsy" cohort comprised temporal lobe epilepsy, genetic generalized epilepsy, and extra temporal epilepsy. We analyzed 7 subcortical and 34 cortical regions (total of 41 brain regions; the mean of left and right structures) that were included in the above specified ENIGMA studies. We extracted the covariate-adjusted Cohen's $d$ standardized mean differences (SMDs) denoting the case versus unaffected comparison subject differences in subcortical volume and cortical thickness/surface area measures. The covariates used in these studies adjusted SMDs for several covariates as indicated in Supplementary Table 1.

\section{Collection of GWAS results among neuropsychiatric disorders}

Publicly available summary statistics from GWAS were downloaded from the Psychiatric Genomics Consortium (PCG) website (https://www.med.unc.edu/pgc/results-anddownloads/) with the exception of GWAS results for MDD coming from an online resource hosted by the University of Edinburgh (https://doi.org/10.7488/ds/2458) and of GWAS results for epilepsy coming from the online Epilepsy Genetic Association Database (epiGAD) (http://www.epigad.org/ gwas_ilae2018_16loci.html). Presented in Supplementary
Table 2 are the numbers of affected cases and unaffected control participants included in each GWAS. Note, the full meta-analysis GWAS of MDD that included data from 23 andMe was not available for public release, thus we used the meta-analysis that combined results from the PGC cohorts and UK Biobank.

\section{Genetic and SMRI phenotype correlations among neuropsychiatric disorders}

Linkage disequilibrium (LD)-score regression, a popular approach designed to analyze summary statistics from GWAS, was used to quantify the amount of shared genetic heritability, or genetic correlation $\left(r_{\mathrm{g}}\right)$, existing between pairs of neuropsychiatric disorders, considering HapMap3 LD-scores [22]. For these analyses, the largest and latest GWAS available for each neuropsychiatric disorder was selected and filtered to exclude markers with INFO $<0.90$ or within the MHC region (hg19:chr6:25-35 Mb) (Supplementary Table 1). GWAS summary statistics were merged with the HapMap3 reference panel (hg37 build), wherein variants with a MAF $\geq 5 \%$ in the HapMap3 dataset were retained, prior to computing (co)heritability estimates.

To derive an estimate of the degree to which sMRI phenotypes were similar among disorders, we computed pairwise Spearman's rank correlation between the Cohen's d SMDs for each pair of disorders. We then used Pearson's correlation to estimate, whether the genetic correlations for each disorder covaried with the sMRI phenotype correlations. We used a traditional permutation framework to generate a null distribution of sMRI phenotype correlations by randomly shuffling Cohen's $d$ values 10,000 times for each pair of disorders, then recalculating sMRI correlations from the shuffled sets. From the null distributions, we derived an empirical permutation $p$ value for each sMRI phenotype correlation. However, a reliable $p$ value could not be calculated due to nonindependence between pairwise caused by sample overlap between imaging studies. Adjustments for sample overlap would be possible with individual-level data, but the present study only had access to summary statistics. In a leave-one-out analysis, we iteratively excluded one pair of disorder correlations from the set and recalculated Spearman's correlation coefficients to determine whether correlations were driven by any pair of disorders. Binomial sign tests were used to determine whether the number of disorders showing the same direction of effect in the sMRI phenotypes was greater than expected by chance (null probability of 50\%). Per brain region, we performed Cochran's $Q$ test implemented in the $R$ package metafor (v.2.1-0) to determine whether variability among Cohen's $\mathrm{d}$ values was greater than expected by chance. All statistical analyses were performed with $R$ version 3.5.2 
Table 1 Sample demographics for the twelve studies by the ENIGMA Consortium into structural brain alterations in neuropsychiatric disorders.

\begin{tabular}{|c|c|c|c|c|c|c|c|c|}
\hline Disorder & MRI measure & Cases $(n)$ & Controls $(n)$ & Total $n$ & Sites & Weighted mean age (cases) & Weighted mean age (controls) & References \\
\hline \multirow[t]{3}{*}{ ADHD } & Cortical thickness & 2246 & 1934 & 4180 & 36 & 19.2 & 18.1 & \multirow[t]{3}{*}[2,28]{} \\
\hline & Surface area & 2246 & 1934 & 4180 & 36 & 19.2 & 18.1 & \\
\hline & Subcortical volume & 1713 & 1529 & 3242 & 23 & 18.6 & & \\
\hline \multirow[t]{3}{*}{ ASD } & Cortical thickness & 1571 & 1651 & 3222 & 49 & 15.4 & & \multirow[t]{3}{*}{ [18] } \\
\hline & Surface area & & & & & & & \\
\hline & Subcortical volume & & & & & & & \\
\hline \multirow[t]{3}{*}{$\mathrm{BD}$} & Cortical thickness & 1837 & 2582 & 4419 & 28 & $38.4^{\mathrm{a}}$ & $35.6^{\mathrm{a}}$ & \multirow[t]{3}{*}[19,30]{} \\
\hline & Surface area & 1820 & 2582 & 4402 & 28 & $38.4^{\mathrm{a}}$ & $35.6^{\mathrm{a}}$ & \\
\hline & Subcortical volume & 1710 & 2594 & 4304 & 20 & $40.1^{\mathrm{a}}$ & $36.5^{\mathrm{a}}$ & \\
\hline \multirow[t]{3}{*}{ Epilepsy } & Cortical thickness & 2149 & 1727 & 3876 & 24 & 34.4 & 33.3 & \multirow[t]{3}{*}{ [7] } \\
\hline & Surface area & & & & & & & \\
\hline & Subcortical volume & & & & & & & \\
\hline \multirow[t]{3}{*}{ MDD } & Cortical thickness & 1911 & 7663 & 9574 & 20 & $44.8^{\mathrm{a}}$ & $54.6^{\mathrm{a}}$ & \multirow[t]{3}{*}[6,22]{} \\
\hline & Surface area & 1902 & 7658 & 9560 & 20 & $44.8^{\mathrm{a}}$ & $54.6^{\mathrm{a}}$ & \\
\hline & Subcortical volume & 1728 & 7199 & 8927 & 15 & $43.3^{\mathrm{a}}$ & $56^{\mathrm{a}}$ & \\
\hline \multirow[t]{3}{*}{ OCD } & Cortical thickness & 1498 & 1435 & 2933 & 27 & 32.1 & 30.5 & \multirow[t]{3}{*}[26,41]{} \\
\hline & Surface area & 1497 & 1433 & 2930 & 27 & 32.1 & 30.5 & \\
\hline & Subcortical volume & 1495 & 1472 & 2967 & 25 & 32.0 & 30.6 & \\
\hline \multirow[t]{3}{*}{ SCZ } & Cortical thickness & 4474 & 5098 & 9572 & 39 & $32.3^{\mathrm{a}}$ & $34.5^{\mathrm{a}}$ & \multirow[t]{3}{*}[27,34]{} \\
\hline & Surface area & 4434 & 5073 & 9507 & 39 & $32.3^{\mathrm{a}}$ & $34.5^{\mathrm{a}}$ & \\
\hline & Subcortical volume & 2028 & 2540 & 4568 & 15 & $34.0^{\mathrm{a}}$ & $31.0^{\mathrm{a}}$ & \\
\hline
\end{tabular}

${ }^{a}$ Weighted mean not provided in paper; computed from descriptive statistics.

(R Core Team, 2018). We adjusted for repeated correlation tests using the Bonferroni procedure. Correlations showing a Bonferroni-adjusted $p<0.05$ were considered significant (threshold $p=0.00227$ ).

\section{Results}

Sample demographics for the twelve studies by the ENIGMA Consortium on structural brain abnormalities in neuropsychiatric disorders are presented in Table 1.

\section{Case-control differences in subcortical volume and cortical surface area and thickness within neuropsychiatric disorders}

Figure 1 presents an anatomical graph of the standardized effect sizes (Cohen's $d$ ) measuring alterations in subcortical volume, cortical surface area and cortical thickness for 41 brain regions within seven neuropsychiatric disordersADHD, ASD, OCD, epilepsy, MDD, BD, and SCZ. These have been reported on prior publications. The variation in color from blue to red illustrates the phenomenon of SBRV, with some regions showing significant reductions (blue) in volume/thickness/surface areas and others not being affected. As indicated by the blueness of the cells, the most prominent reductions were seen for SCZ (mean Cohen's d across all regions $=-0.22, \mathrm{SE}=0.014$ ), epilepsy (mean Cohen's $d=-0.12, \mathrm{SE}=0.017$ ) and $\mathrm{BD}$ (mean Cohen's $d=-0.097, \mathrm{SE}=0.011)$. The smallest changes were observed for MDD (mean Cohen's $d=-0.018$, SE $=$ 0.006). All regions except for the caudate and putamen exhibited significant differences in the magnitude of Cohen's d across disorders (Cochran's Q $p$ values $=$ $0.012-2.8 \times 10^{-32}$ ). Eighteen sMRI phenotypes exhibited homogeneity with respect to sign of Cohen's $d$ across each of the neuropsychiatric disorders evaluated (binomial sign test $p$ values $<0.05$ ): cortical thicknesses for caudal middle frontal gyrus, entorhinal cortex, fusiform gyrus, inferior temporal gyrus, insula, lateral orbitofrontal cortex, lingual gyrus, middle temporal gyrus, paracentral lobule, parahippocampal gyrus, pars opercularis of inferior temporal gyrus, precentral gyrus, precuneus, rostral anterior cingulate cortex, and supramarginal gyrus; subcortical volume for the hippocampus; and surface area for middle temporal gyrus, pars triangularis of inferior temporal gyrus, and pericalcarine cortex. For sMRI phenotypes for 39 regions of interest varying degrees of heterogeneity were noted in terms of discrepancy of signs of Cohen's $d$. For example, individuals with ASD showed a slightly thicker cortex in the rostral 


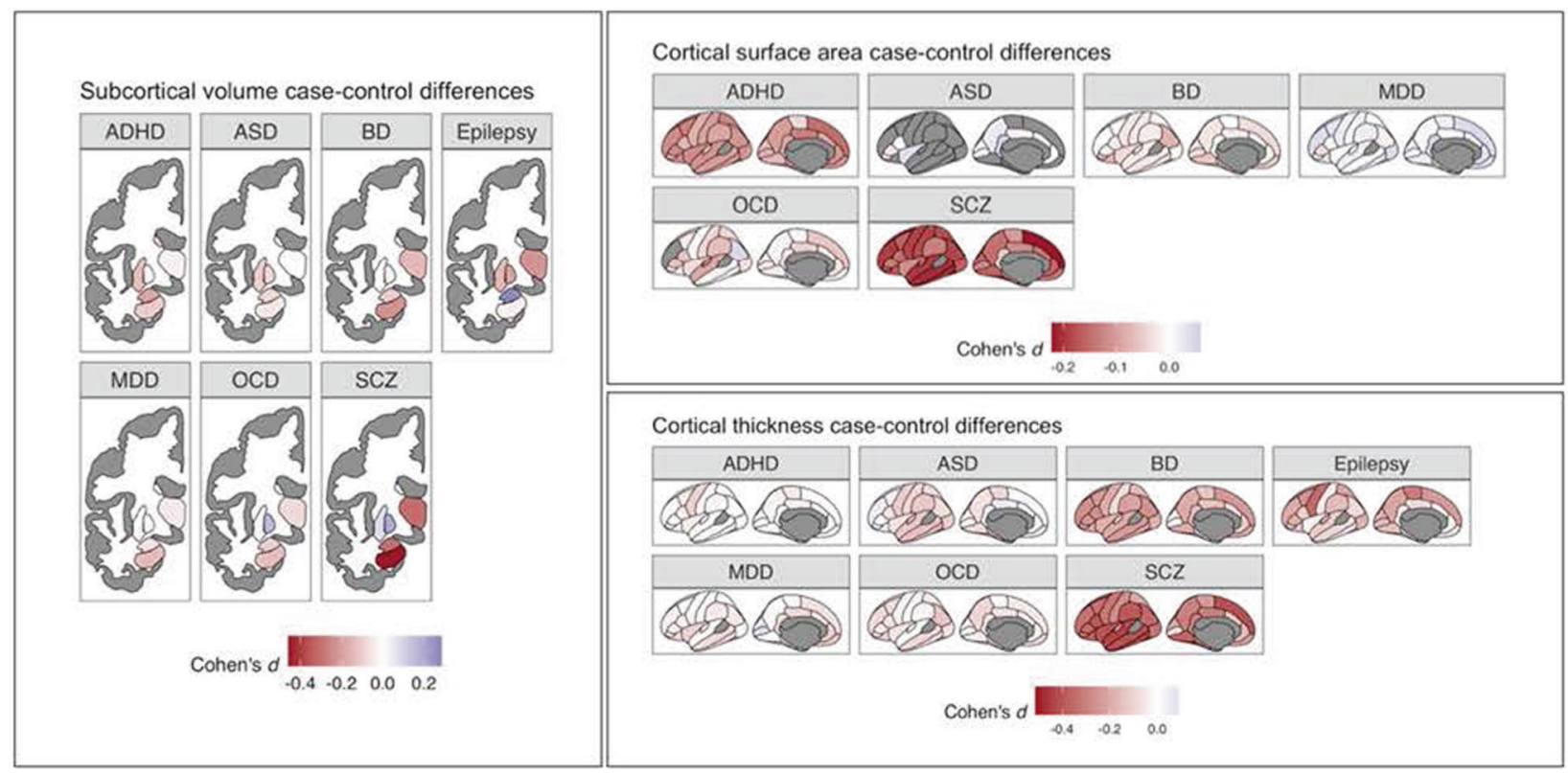

Fig. 1 A brain imaging plot showing standardized mean differences (Cohen's $d$ ) measuring case-control differences in subcortical volumes and cortical thickness for seven neuropsychiatric disorders. Results were obtained from ENIGMA working group publications. Negative values for Cohen's $d$ indicate smaller sizes of

middle frontal gyrus, individuals with ADHD showed no difference, and all other disorders showed a thinner cortex in this region compared to controls.

\section{sMRI phenotype correlations among neuropsychiatric disorders}

For each pair of disorders, we computed the Pearson correlation between their sMRI phenotypes listed in Fig. 1. These are listed in Table 2 (and visualized in Fig. 2), sorted by the magnitude of the correlation. The $p$ values reported in Table 2 are potentially downwardly biased due to inability to properly adjust for spatial coherence of nearby brain regions. Traditional permutation $p$ values are provided as a column in Table 2, which attempts to correct for potential biases due to spatial coherence. However, we were restricted from using a spatial permutation framework to generate a null distribution of correlations, because we are jointly analyzing two cortical maps (cortical thickness and surface area) that are fully overlapped. The highest positive correlation was between $\mathrm{SCZ}$ and BD $\left(r=0.81, \mathrm{df}=73, p<1.3 \times 10^{-18}\right.$, Bonferroni $p=2.38 \times 10^{-17}$ ). There were a few additional nominally significant negative correlations, which did not survive multiple testing correction: MDD and epilepsy $(r=-0.37, p=$ 0.02), MDD and ADHD $(r=-0.33, p=0.004), \mathrm{SCZ}$ and ADHD $(r=-0.32, p=0.005)$, ADHD and epilepsy $(r=$ $-0.36, p=0.02)$, and a positive correlation between MDD and $\operatorname{ASD}(r=0.26, p=0.02)$. brain regions in cases versus unaffected comparisons. Note: ADHD attention-deficit/hyperactivity disorder, ASD autism spectrum disorder, BD bipolar disorder, MDD major depressive disorder, OCD obsessive compulsive disorder, SCZ schizophrenia.

\section{Correlation of shared genetic heritability with brain structural correlation}

Figure 3 shows the pairwise correlations of sMRI phenotypes and genetic overlap across each pair of neuropsychiatric disorders. The LD-score cross-disorder genetic correlations are positively correlated with the sMRI phenotype cross-disorder correlations (Spearman's $\rho=$ $0.44, p=0.049$ ). Leave-one-out sensitivity analyses confirmed that the direction of the correlation was positive and remained moderate in magnitude despite removal of individual pairs of disorders from the correlation test (range of Spearman's $\rho=0.35-0.58$ ), except for removing SCZ/BD (Spearman's $\rho=0.35$ ). SCZ and BD showed the highest degree of concordance with respect to genetic and sMRI phenotype correlations.

\section{Discussion}

Our analysis of summary statistics from the ENIGMA ADHD, ASD, BD, MDD, OCD, SCZ, and epilepsy Working Groups and the predominantly PGC case-control GWAS identified two novel findings. First, we found substantial correlations for some disorders in the pattern of sMRI case-control differences across subcortical and cortical regions in line with recently published study of [23]. Second, these cross-disorder 
Table 2 Cross-disorder structural MRI phenotype correlations (ordered from smallest to largest $p$ value) based on Cohen's $d$ values obtained from the ENIGMA Project.

\begin{tabular}{llllllll}
\hline Disorder 1 & Disorder 2 & $\begin{array}{l}\text { sMRI correlation } \\
\text { Pearson's } r\end{array}$ & df & se & $p$ value & $\begin{array}{l}\text { Boferroni } \\
\text { adjusted } p \text { value }\end{array}$ & $\begin{array}{l}\text { Permutation } \\
p \text { value }\end{array}$ \\
\hline BD & SCZ & 0.81 & 73 & 0.068 & $1.13 \mathrm{E}-18$ & $2.38 \mathrm{E}-17$ & $<1 \mathrm{E} 10-4$ \\
BD & MDD & 0.69 & 73 & 0.085 & $1.21 \mathrm{E}-11$ & $2.55 \mathrm{E}-10$ & $<1 \mathrm{E} 10-4$ \\
OCD & SCZ & 0.65 & 72 & 0.09 & $5.53 \mathrm{E}-10$ & $1.16 \mathrm{E}-08$ & $<1 \mathrm{E} 10-4$ \\
MDD & SCZ & 0.58 & 73 & 0.095 & $5.55 \mathrm{E}-08$ & $1.17 \mathrm{E}-06$ & $<1 \mathrm{E} 10-4$ \\
ADHD & BD & -0.53 & 73 & 0.099 & $1.18 \mathrm{E}-06$ & $2.48 \mathrm{E}-05$ & $<1 \mathrm{E} 10-4$ \\
BD & OCD & 0.5 & 72 & 0.102 & $4.74 \mathrm{E}-06$ & $9.95 \mathrm{E}-05$ & $<1 \mathrm{E} 10-4$ \\
MDD & OCD & 0.46 & 72 & 0.104 & $3.28 \mathrm{E}-05$ & $6.89 \mathrm{E}-04$ & $<1 \mathrm{E} 10-4$ \\
ASD & BD & 0.38 & 73 & 0.108 & $8.98 \mathrm{E}-04$ & 0.02 & $<1 \mathrm{E} 10-4$ \\
ASD & SCZ & 0.36 & 73 & 0.109 & $1.35 \mathrm{E}-03$ & 0.03 & 0.0176 \\
ADHD & MDD & -0.33 & 73 & 0.111 & $4.27 \mathrm{E}-03$ & 0.09 & 0.019 \\
ADHD & SCZ & -0.32 & 73 & 0.111 & $4.63 \mathrm{E}-03$ & 0.1 & 0.0014 \\
Epilepsy & MDD & -0.37 & 39 & 0.149 & 0.02 & 0.38 & 0.0056 \\
ADHD & Epilepsy & -0.36 & 39 & 0.149 & 0.02 & 0.41 & 0.004 \\
ASD & MDD & 0.26 & 73 & 0.113 & 0.02 & 0.46 & 0.024 \\
Epilepsy & OCD & -0.19 & 39 & 0.157 & 0.23 & 1 & 0.22 \\
BD & Epilepsy & 0.17 & 39 & 0.158 & 0.3 & 1 & 0.3 \\
ADHD & OCD & -0.1 & 72 & 0.117 & 0.39 & 1 & 0.39 \\
ADHD & ASD & -0.06 & 73 & 0.117 & 0.6 & 1 & 0.6 \\
Epilepsy & SCZ & -0.03 & 39 & 0.16 & 0.86 & 1 & 0.85 \\
ASD & Epilepsy & 0.02 & 39 & 0.16 & 0.91 & 1 & 0.91 \\
ASD & OCD & 0 & 72 & 0.118 & 0.97 & 1 & 0.97 \\
\hline & & & & & & & 1 \\
\hline
\end{tabular}

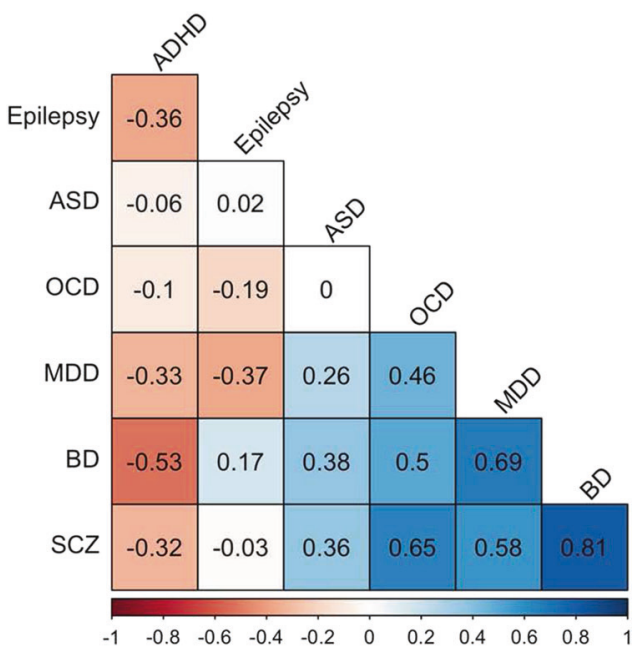

Fig. 2 A heatmap of the cross-disorder pairwise sMRI correlations between seven neuropsychiatric disorders examined in this study. Colors in the plot correspond to the magnitude of the Pearson's $r$ coefficients, which are provided in each tile. Note: ADHD attentiondeficit/hyperactivity disorder, ASD autism spectrum disorder, BD bipolar disorder, MDD major depressive disorder, OCD obsessive compulsive disorder, SCZ schizophrenia.

correlations in SBRV could partly be explained by the genetic correlations reported for these disorders from GWAS [3].

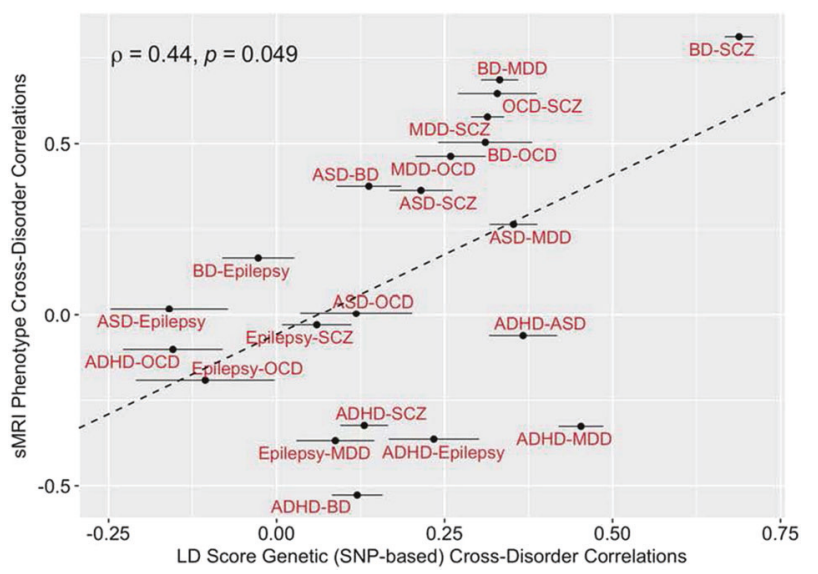

Fig. 3 Scatter plot showing the correlation of correlations. Genetic correlations (rg) computed by LD-score regression are on the horizontal axis (with standard error bars), with correlations of Cohen's $d$ values displayed on the vertical axis. Each dot is color-coded according to the pairwise disorder correlations that were computed. The dotted line represents the best-fit regression line. The Spearman's rho $(\rho)$ and $p$ value are provided at the top-left corner of the panel. Note: ADHD attention-deficit/hyperactivity disorder, ASD autism spectrum disorder, BD bipolar disorder, MDD major depressive disorder, OCD obsessive compulsive disorder, SCZ schizophrenia.

The cross-disorder correlations in SBRV are intriguing because, like cross-disorder genetic correlations, they suggest that these disorders, to varying degrees, share aspects 
of their etiology and pathophysiology. Any interpretation of the cross-disorder sMRI correlations must keep in mind that, for all disorders, the case-control differences in SMRI measures are small (Fig. 1). The largest Cohen's $d$ values are only -0.5 for SCZ [20, 21], -0.4 for epilepsy [15], -0.3 for BD $[13,14],-0.2$ for ADHD [10, 11] and ASDs [12], and -0.1 for MDD [16, 17] and OCD [18, 19]. These small case-control differences are consistent with results from GWAS and environmental risk studies, which speaks to the fact that the effects of common risk factors are, with some rare exceptions, individually small. Although it is conceivable that these small risks could accumulate to create a more dramatic pathophysiology in the brain, the ENIGMA data show that this is not the case for sMRI measures. Consistent with this finding, interindividual differences in neuroimaging account for only a small amount of the variance in symptom expression or behavioral measures of symptomatic or behavioral variance [24].

The most prominent case-control differences in cortical thickness/surface area and subcortical volumes were observed for SCZ [20, 21] and BD [13, 14]. These disorders also had the highest sMRI phenotype correlations and both also showed strong sMRI phenotype correlations with MDD [16, 17] and OCD [18, 19]. As Fig. 2 shows, these disorders clustered together in the three-dimensional configuration required to capture cross-disorder sMRI phenotype similarity. The high sMRI correlation between SCZ and BD is consistent with prior reports of SMRI similarities between the two disorders [25]. Moreover, a large body of literature reports substantial etiologic overlap between the two disorders [26-30]. Because of such data, the SCZ and $\mathrm{BD}$ have been described as sharing a continuum of etiology leading to psychotic [31], neurophysiological [31] and neurocognitive [32] symptoms. The ENPACT study [33] showed shared fronto-temporo-occipital gray matter volume deficits in the right hemisphere of two disorders. A systematic review of associations between functional MRI activity and polygenic risk for SCZ and BD [26] reported that genetic load for these disorders affects task-related recruitment of predominantly frontal lobe brain regions.

Many studies have reported that OCD can be a comorbid diagnosis with SCZ or that patients with SCZ can have OCD symptoms [34-41]. Presented findings of a significant overlap in sMRI phenotypes along with the known SCZ/OCD genetic correlations suggests that more work should examine shared pathophysiologic features between these disorders and should assess the degree to which confounds, such as medication status or chronicity, might explain these results.

The sMRI phenotype correlations mirror, to some extent, the cross-disorder correlations from GWAS. Figure 3 shows a modest, yet distinct, linear correlation between the sMRI phenotype and genetic correlations. In the upper right-hand section of the plot, we see disorders having high genetic and high sMRI correlations. These are SCZ/BD, SZ/MDD, BD/ MDD, OCD/BD, and OCD/MDD. The inclusion of MDD in this group is notable given that it is part of the bipolar diagnosis and often occurs comorbid with other disorders. MDD also has a high genetic correlation with ADHD but a negative SMRI correlation, which makes that pair an outlier in Fig. 3.

In the lower left region of Fig. 3, we see disorders with low genetic and low sMRI correlations. These involve correlations of epilepsy, and correlations of ADHD with all disorders except ASDs and MDD, although the latter is somewhat of an outlier. ASDs tend to have both modest genetic correlations and modest sMRI correlations with most other disorders and, hence, populates the middle range of the figure. Like the sMRI correlations among disorders, all genetic correlations with epilepsy are low, which is consistent with the low genetic correlation between neurological and psychiatric disorders as reported by [2].

The finding that SBRV correlations are correlated with genetic correlations suggests that future studies of SBRV should consider genetic sources of etiology. Yet, because only about $24 \%$ of the variance in the SBRV correlations can be accounted for by the genetic correlations, environmental sources of etiology and disease-specific genetic contributions must also be considered. These include shared confounders, such as chronicity and medication exposure, along with shared etiologic events such as birth complications or exposure to toxins in utero. Our prior studies of SBRV in ADHD implicated the regulation of genes in apoptosis, autophagy and neurodevelopment pathways in ADHD [42, 43]. Neurodevelopmental pathways had also been implicated in the cross-disorder analysis of the PCG [3], which suggests that cross-disorder similarities in these pathways may account for cross-disorder similarities in SBRV.

Although we used data derived from very large samples (ENIGMA, iPSYCH, and the PGC), several limitations moderate the strength of our conclusions. We inherit all the limitations of the constituent studies, but are further limited because we analyzed summary statistics, not the original data, which would require the sharing of individual subject level data, an ongoing effort among the ENIGMA disorder working groups. Thus, we cannot determine whether the possible use of controls shared among studies affected our results. It is also possible that some research participants were included in the genetic and sMRI data sets for the same disorder. The $p$ value obtained by our Spearman's correlation test of cross-disorder sMRI and genetic correlations may be inaccurate due to spatial autocorrelation among sMRI Cohen's $d$ estimates, which can downwardly bias standard errors and lead to deflated $p$ values. Considering we are not able to completely address with autocorrelation among brain regions using summary statistics alone, the $p$ value from our primary analysis (presented in 
Fig. 3) should be interpreted with caution. Another problem is that we could not address effects of medications or chronicity on brain structure. Furthermore, for some of the disorders, we could use youth and adult data, whereas for others only adult effect data were used. Because findings can differ substantially depending on the age range of the samples included (e.g., [10, 11, 18, 19], this might have influenced our findings. For these reasons, analyses of participant level data will be needed to address these issues to draw stronger and more detailed conclusions. We also did not have any longitudinal data available, which limits the ability to test hypotheses about shared and unique developmental trajectories among disorders.

Despite these limitations, we have documented crossdisorder correlations in SBRV as assessed by sMRI. These cross-disorder SBRV correlations are positively associated with the disorders' corresponding cross-disorder genetic correlations. This finding is a novel contribution worthy of further study that contributes to novel body of literature focused on cross-level correspondence of genetic and neuroimaging presentations of different psychiatric disorders [44-49]. Our work supports conclusions from previous GWAS studies suggesting a partially shared etiology and pathophysiology among many disorders $[2,50]$. Disorders like SCZ and BD or ADHD and ASD, which are distinct in the diagnostic nomenclature, show significant overlap in etiology and pathophysiology. Further studies are needed to discern why brain regions are selectively affected by the risk factors that cause sMRI abnormalities [42, 43] and why these effects are correlated across disorders. Such studies may give insights into new treatment targets.

\section{Data availability}

URLs for GWAS SCZ from ckqny.scz2snpres.gz (https://www.med.unc.edu/pgc/results-and-downloads), ASD from iPSYCH-PGC_ASD_Nov2017.gz (https://www.med. unc.edu/pgc/results-and-downloads), OCD from PGC_OCD_Aug2017-20171122T182645Z-001.zip > ocd_aug2017.gz (https://www.med.unc.edu/pgc/results-and-downloads),

ADHD from adhd_ul2017.gz (https://www.med.unc.edu/pgc/ results-and-downloads), BD from daner_PGC_BIP32b_mds7a_0416a.gz (https://www.med.unc.edu/pgc/results-and-dow nloads), Epilepsy from all_epilepsy_METAL.gz (http://www. epigad.org/gwas_ilae2018_16loci.html), and MDD from PGC_UKB_depression_genome-wide.txt (https://doi.org/10. 7488/ds/2458).

\section{Code availability}

Custom $R$ scripts used to generate results in this study can be made available upon request.
Acknowledgements SVF is supported by the European Union's Seventh Framework Program for research, technological development and demonstration under grant agreement no. 602805, the European Union's Horizon 2020 research and innovation program under grant agreements nos. 667302 and 728018 and NIMH grants 5R01MH101519 and U01 MH109536-01. Research Council of Norway (\#223273). BF is supported by a personal Vici grant from the Netherlands Organization for Scientific Research (NWO, grant number 91813669) and by a grant from the European Union's Horizon 2020 program for the CoCa project (grant agreement no 667302). ENIGMA work is supported by NIH grants U54 EB020403 (PI: PT), R01 MH116147 (PI: PT) and R01MH117601 (MPIs: NJ and LS). MH is supported by a personal Veni grant from the Netherlands Organization for Scientific Research (NWO, grant number 91619115). CMD is supported by NIH grants R01 NS065838 and R21 NS107739. PR is a recipient of a pre-doctoral fellowship from the Agència de Gestió d'Ajuts Universitaris i de Recerca (AGAUR), Generalitat de Catalunya, Spain (2016FI_B 00899). LS is supported by a NHMRC Career Development Fellowship (1140764). SMS is supported by Epilepsy Society, UK, and the work was partly undertaken at UCLH/UCL, which received a proportion of funding from the UK Department of Health's NIHR Biomedical Research Centers funding scheme.). TGMVE is supported by NIH grants U54 EB020403 (PI: PT), R01 MH116147 (PI: PT), R01MH117601 (MPIs: NJ and LS), and R01MH121246 (MPIs: Turner, TGMVE, and Calhoun).

\section{Compliance with ethical standards}

Conflict of interest OA has received speaker's honorarium from Lundbeck and is a consultant to HealthLytix. In the past year, SVF received income, potential income, travel expenses continuing education support, and/or research support from Tris, Otsuka, Arbor, Ironshore, Shire, Akili Interactive Labs, Enzymotec, Sunovion, Supernus and Genomind. With his institution, he has US patent US20130217707 A1 for the use of sodium-hydrogen exchange inhibitors in the treatment of ADHD. He also receives royalties from books published by Guilford Press: Straight Talk about Your Child's Mental Health, Oxford University Press: Schizophrenia: The Facts and Elsevier: ADHD: Non-Pharmacologic Interventions. He is Program Director of www.adhdinadults.com. BF received educational speaking fees from Medice. All other authors declare no conflict of interest.

Publisher's note Springer Nature remains neutral with regard to jurisdictional claims in published maps and institutional affiliations.

Open Access This article is licensed under a Creative Commons Attribution 4.0 International License, which permits use, sharing, adaptation, distribution and reproduction in any medium or format, as long as you give appropriate credit to the original author(s) and the source, provide a link to the Creative Commons license, and indicate if changes were made. The images or other third party material in this article are included in the article's Creative Commons license, unless indicated otherwise in a credit line to the material. If material is not included in the article's Creative Commons license and your intended use is not permitted by statutory regulation or exceeds the permitted use, you will need to obtain permission directly from the copyright holder. To view a copy of this license, visit http://creativecommons. org/licenses/by/4.0/.

\section{References}

1. Polderman TJ, Benyamin B, de Leeuw CA, Sullivan PF, van Bochoven A, Visscher PM, et al. Meta-analysis of the heritability 
of human traits based on fifty years of twin studies. Nat Genet. 2015;47:702-9. https://doi.org/10.1038/ng.3285.

2. Anttila V, Bulik-Sullivan B, Finucane HK, Walters RK, Bras J, Duncan L, et al. Analysis of shared heritability in common disorders of the brain. Science. 2018;360:eaap8757. https://doi.org/ 10.1126/science.aap8757.

3. Lee PH, Anttila V, Won H, Feng Y-CA, Rosenthal J, Zhu Z, et al. Genome wide meta-analysis identifies genomic relationships, novel loci, and pleiotropic mechanisms across eight psychiatric disorders. bioRxiv. 2019. https://doi.org/10.1101/528117.

4. Lee SH, Ripke S, Neale BM, Faraone SV, Purcell SM, Perlis RH, et al. Genetic relationship between five psychiatric disorders estimated from genome-wide SNPs. Nat Genet. 2013;45:984-94. https://doi.org/10.1038/ng.2711.

5. Smoller JW, Andreassen OA, Edenberg HJ, Faraone SV, Glatt SJ, Kendler KS. Correction to: psychiatric genetics and the structure of psychopathology. Mol Psychiatry. 2018a. https://doi.org/10. 1038/s41380-018-0026-4.

6. Buckholtz JoshuaW, Meyer-Lindenberg A. Psychopathology and the human connectome: toward a transdiagnostic model of risk for mental illness. Neuron. 2012;74:990-1004. https://doi.org/10. 1016/j.neuron.2012.06.002.

7. Brouwer RM, Panizzon MS, Glahn DC, Hibar DP, Hua X, Jahanshad N, et al. Genetic influences on individual differences in longitudinal changes in global and subcortical brain volumes: results of the ENIGMA plasticity working group. Hum Brain Mapp. 2017;38:4444-58. https://doi.org/10.1002/hbm.23672.

8. Ge T, Chen C-Y, Neale BM, Sabuncu MR, Smoller JW. Phenome-wide heritability analysis of the UK Biobank. PLOS Genetics 2017;13:e1006711.

9. Thompson PM, Andreassen OA, Arias-Vasquez A, Bearden CE, Boedhoe PS, Brouwer RM, et al. ENIGMA and the individual: Predicting factors that affect the brain in 35 countries worldwide. NeuroImage. 2017;145:389-408. https://doi.org/10.1016/ j.neuroimage.2015.11.057.

10. Hoogman M, Bralten J, Hibar DP, Mennes M, Zwiers MP, Schweren LSJ, et al. Subcortical brain volume differences in participants with attention deficit hyperactivity disorder in children and adults: a cross-sectional mega-analysis. Lancet Psychiatry. 2017;4:310-9. https://doi.org/10.1016/S2215-0366(17) 30049-4.

11. Hoogman M, Muetzel R, Guimaraes JP, Shumskaya E, Mennes M, Zwiers MP, et al. Brain imaging of the cortex in ADHD: a coordinated analysis of large-scale clinical and population-based samples. Am J Psychiatry. 2019. https://doi.org/10.1176/appi.ajp. 2019.18091033.

12. van Rooij D, Anagnostou E, Arango C, Auzias G, Behrmann M, Busatto GF, et al. Cortical and subcortical brain morphometry differences between patients with autism spectrum disorder and healthy individuals across the lifespan: results from the ENIGMA ASD Working Group. Am J Psychiatry. 2018;175:359-69. https:// doi.org/10.1176/appi.ajp.2017.17010100.

13. Hibar DP, Westlye LT, Doan NT, Jahanshad N, Cheung JW, Ching CRK, et al. Cortical abnormalities in bipolar disorder: an MRI analysis of 6503 individuals from the ENIGMA Bipolar Disorder Working Group. Mol Psychiatry. 2017;23:932. https:// doi.org/10.1038/mp.2017.73.

14. Hibar DP, Westlye LT, van Erp TG, Rasmussen J, Leonardo CD, Faskowitz J, et al. Subcortical volumetric abnormalities in bipolar disorder. Mol Psychiatry. 2016;21:1710-6. https://doi.org/10. 1038/mp.2015.227.

15. Whelan CD, Altmann A, Botia JA, Jahanshad N, Hibar DP, Absil $\mathrm{J}$, et al. Structural brain abnormalities in the common epilepsies assessed in a worldwide ENIGMA study. Brain. 2018;141:391-408. https://doi.org/10.1093/brain/awx341.
16. Schmaal L, Hibar DP, Sämann PG, Hall GB, Baune BT, Jahanshad N, et al. Cortical abnormalities in adults and adolescents with major depression based on brain scans from 20 cohorts worldwide in the ENIGMA Major Depressive Disorder Working Group. Mol Psychiatry. 2016;22:900. https://doi.org/10.1038/mp.2016.60.

17. Schmaal L, Veltman DJ, van Erp TGM, Sämann PG, Frodl T, Jahanshad N, et al. Subcortical brain alterations in major depressive disorder: findings from the ENIGMA Major Depressive Disorder working group. Mol Psychiatry. 2015;21:806. https:// doi.org/10.1038/mp.2015.69.

18. Boedhoe PSW, Schmaal L, Abe Y, Alonso P, Ameis SH, Anticevic A, et al. Cortical abnormalities associated with pediatric and adult obsessive-compulsive disorder: findings from the ENIGMA obsessive-compulsive disorder working group. Am J Psychiatry. 2018;175:453-62. https://doi.org/10.1176/appi.ajp. 2017.17050485.

19. Boedhoe PSW, Schmaal L, Abe Y, Ameis SH, Arnold PD, Batistuzzo MC, et al. Distinct subcortical volume alterations in pediatric and adult OCD: a worldwide meta- and mega-analysis. Am J Psychiatry. 2017;174:60-9. https://doi.org/10.1176/appi.ajp. 2016.16020201.

20. van Erp TGM, Hibar DP, Rasmussen JM, Glahn DC, Pearlson GD, Andreassen OA, et al. Subcortical brain volume abnormalities in 2028 individuals with schizophrenia and 2540 healthy controls via the ENIGMA consortium. Mol Psychiatry. 2015;21:547. https://doi.org/10.1038/mp.2015.63.

21. van Erp TGM, Walton E, Hibar DP, Schmaal L, Jiang W, Glahn DC, et al. Cortical brain abnormalities in 4474 individuals with schizophrenia and 5098 control subjects via the enhancing neuro imaging genetics through meta analysis (ENIGMA) consortium. Biol Psychiatry. 2018;84:644-54. https://doi.org/10.1016/j. biopsych.2018.04.023.

22. Bulik-Sullivan BK, Loh PR, Finucane HK, Ripke S, Yang J, Schizophrenia Working Group of the Psychiatric Genomics, C, et al. LD score regression distinguishes confounding from polygenicity in genome-wide association studies. Nat Genet. 2015;47:291-5. https://doi.org/10.1038/ng.3211.

23. Opel N, Goltermann J, Hermesdorf M, Berger K, Baune BT, Dannlowski U. Cross-Disorder Analysis of Brain Structural Abnormalities in Six Major Psychiatric Disorders: A Secondary Analysis of Mega- and Meta-analytical Findings From the ENIGMA Consortium. Biol Psychiatry. 2020.

24. Paulus MP, Thompson WK. The Challenges and Opportunities of Small Effects: The New Normal in Academic Psychiatry. JAMA Psychiatry. 2019;76:353-4. https://doi.org/10.1001/jamapsychia try.2018.4540.

25. Rimol LM, Hartberg CB, Nesvag R, Fennema-Notestine C, Hagler DJ Jr, Pung CJ, et al. Cortical thickness and subcortical volumes in schizophrenia and bipolar disorder. Biol Psychiatry. 2010;68:41-50. https://doi.org/10.1016/j.biopsych.2010.03.036.

26. Dezhina Z, Ranlund S, Kyriakopoulos M, Williams SCR, Dima D. A systematic review of associations between functional MRI activity and polygenic risk for schizophrenia and bipolar disorder. Brain Imaging Behav. 2019;13:862-77. https://doi.org/10.1007/ s11682-018-9879-z.

27. Haukvik UK, Tamnes CK, Soderman E, Agartz I. Neuroimaging hippocampal subfields in schizophrenia and bipolar disorder: a systematic review and meta-analysis. J Psychiatr Res. 2018;104: 217-26. https://doi.org/10.1016/j.jpsychires.2018.08.012.

28. Berrettini W. Bipolar disorder and schizophrenia: convergent molecular data. Neuromolecular Med. 2004;5:109.

29. Lizano P, Bannai D, Lutz O, Kim LA, Miller J, Keshavan M. A Meta-analysis of retinal cytoarchitectural abnormalities in schizophrenia and bipolar disorder. Schizophr Bull. 2019. https://doi. org/10.1093/schbul/sbz029. 
30. Bora E, Akgul O, Ceylan D, Ozerdem A. Neurological soft signs in bipolar disorder in comparison to healthy controls and schizophrenia: a meta-analysis. Eur Neuropsychopharmacol. 2018;28: 1185-93. https://doi.org/10.1016/j.euroneuro.2018.08.006.

31. Thaker G. Psychosis endophenotypes in schizophrenia and bipolar disorder. Schizophr Bull. 2008;34:720-1. https://doi.org/10.1093/ schbul/sbn055.

32. Kim D, Kim J, Koo T, Yun H, Won S. Shared and Distinct Neurocognitive Endophenotypes of Schizophrenia and Psychotic Bipolar Disorder. Clin Psychopharmacol Neurosci. 2015;13: 94-102.

33. Maggioni E, Crespo-Facorro B, Nenadic I, Benedetti F, Gaser C, Sauer $\mathrm{H}$, et al. Common and distinct structural features of schizophrenia and bipolar disorder: The European Network on Psychosis, Affective disorders and Cognitive Trajectory (ENPACT) study. PLoS ONE. 2017;12:e0188000. https://doi.org/10.1371/ journal.pone. 0188000 .

34. Grover S, Sahoo S, Surendran I. Obsessive-compulsive symptoms in schizophrenia: a review. Acta Neuropsychiatr. 2019;31:63-73. https://doi.org/10.1017/neu.2018.27.

35. Hwang MLO. Schizophrenia with obsessive-compulsive features: assessment and treatment. Psychiatr Ann. 1994;24:468-72. https://doi.org/10.3928/0048-5713-19940901-08.

36. Özdemir Ö, Tükel R, Türksoy N, Üçok A. Clinical characteristics in obsessive-compulsive disorder with schizophrenia. Compr Psychiatry. 2003;44:311-6. https://doi.org/10.1016/S0010-440X (03)00093-2.

37. Poyurovsky M, Zohar J, Glick I, Koran LM, Weizman R, Tandon $\mathrm{R}$, et al. Obsessive-compulsive symptoms in schizophrenia: implications for future psychiatric classifications. Compr Psychiatry. 2012;53:480-3. https://doi.org/10.1016/j.comppsych. 2011.08.009.

38. Schirmbeck F, Zink M. Comorbid obsessive-compulsive symptoms in schizophrenia: contributions of pharmacological and genetic factors. Front Pharmacol. 2013;4:99. https://doi.org/10. 3389/fphar.2013.00099.

39. Swets M, Dekker J, van Emmerik-van Oortmerssen K, Smid GE, Smit F, de Haan L, et al. The obsessive compulsive spectrum in schizophrenia, a meta-analysis and meta-regression exploring prevalence rates. Schizophr Res. 2014;152:458-68. https://doi. org/10.1016/j.schres.2013.10.033.

40. Tumkaya S, Karadag F, Oguzhanoglu NK, Tekkanat C, Varma G, Ozdel O, et al. Schizophrenia with obsessive-compulsive disorder and obsessive-compulsive disorder with poor insight: a neuropsychological comparison. Psychiatry Res. 2009;165:38-46. https://doi.org/10.1016/j.psychres.2007.07.031.

41. Cunill R, Castells X, Simeon D. Relationships between obsessivecompulsive symptomatology and severity of psychosis in schizophrenia: a systematic review and meta-analysis. J Clin Psychiatry. 2009;70:70-82. https://doi.org/10.4088/jcp.07r03618.

42. Hess JL, Akutagava-Martins GC, Patak JD, Glatt SJ, Faraone SV. Why is there selective subcortical vulnerability in ADHD? Clues from postmortem brain gene expression data. Mol Psychiatry. 2017. https://doi.org/10.1038/mp.2017.242.

43. Hess JL, Radonjić NV, Patak J, Glatt SJ, Faraone SV (2019). Spatial organization of cells and variable expression of autophagy, apoptosis, and neurodevelopmental genes might underlie selective brain region vulnerability in Attention-Deficit/Hyperactivity Disorder. bioRxiv. 2019. https://doi.org/10.1101/652792.

44. Gandal MJ, Haney JR, Parikshak NN, Leppa V, Ramaswami G, Hartl C, et al. Shared molecular neuropathology across major psychiatric disorders parallels polygenic overlap. Science. 2018;359:693.

45. Anderson KM, Collins MA, Kong R, Fang K, Li J, He T, et al. Convergent molecular, cellular, and neural signatures of major depressive disorder. bioRxiv 2020: https://doi.org/10.1101/2020. 02.10.942227.

46. Romme IA, de Reus MA, Ophoff RA, Kahn RS, van den Heuvel MP. Connectome Disconnectivity and Cortical Gene Expression in Patients With Schizophrenia. Biol Psychiatry. 2017;81: 495-502.

47. Seidlitz J, Nadig A, Liu S, Bethlehem RAI, Vértes PE, Morgan SE, et al. Transcriptomic and cellular decoding of regional brain vulnerability to neurogenetic disorders. Nat Commun. 2020;11:3358

48. Goodkind M, Eickhoff SB, Oathes DJ, Jiang Y, Chang A, JonesHagata LB, et al. Identification of a Common Neurobiological Substrate for Mental Illness. JAMA Psychiatry. 2015;72:305-15.

49. Disorder WCftA-DH, Disorder AS, Disorder B, Disorder MD, Disorder O-C, Groups aSEW. Virtual Histology of Cortical Thickness and Shared Neurobiology in 6 Psychiatric Disorders. JAMA Psychiatry. 2020.

50. Smoller JW, Andreassen OA, Edenberg HJ, Faraone SV, Glatt SJ, Kendler KS. Psychiatric genetics and the structure of psychopathology. Mol Psychiatry. 2018b. https://doi.org/10.1038/ s41380-017-0010-4.

\section{Affiliations}

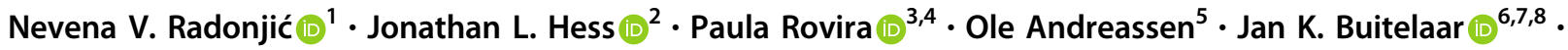
Christopher R. K. Ching ${ }^{9} \cdot$ Barbara Franke $\mathbb{D I D}^{7,10,11} \cdot$ Martine Hoogman $^{10} \cdot$ Neda Jahanshad $^{12}{ }^{12} \cdot$ Carrie McDonald $^{13}$. Lianne Schmaal $\mathbb{1}^{14,15}$. Sanjay M. Sisodiya ${ }^{16,17} \cdot$ Dan J. Stein $\mathbb{1}^{18}$. Odile A. van den Heuvel ${ }^{19}$. Theo G. M. van Erp $\mathbb{1}^{20,21} \cdot$ Daan van Rooij ${ }^{22} \cdot$ Dick J. Veltman ${ }^{19} \cdot$ Paul Thompson ${ }^{23} \cdot$ Stephen V. Faraone $\mathbb{(}^{2}$

1 Department of Psychiatry, SUNY Upstate Medical University, Syracuse, NY, USA

2 Departments of Psychiatry and of Neuroscience and Physiology, SUNY Upstate Medical University, Syracuse, NY, USA

3 Psychiatric Genetics Unit, Group of Psychiatry, Mental Health and Addiction, Vall d'Hebron Research Institute (VHIR), Universitat Autònoma de Barcelona, Barcelona, Spain

4 Department of Psychiatry, Hospital Universitari Vall d'Hebron, Barcelona, Spain
5 NORMENT_-Institute of Clinical Medicine, Division of Mental Health and Addiction, Oslo University Hospital, University of Oslo, Oslo, Norway

6 Radboudumc, Radboud University Medical Center, Nijmegen, The Netherlands

7 Donders Institute for Brain, Cognition and Behaviour, Radboud University Medical Center, Nijmegen, The Netherlands

8 Department of Cognitive Neuroscience, Radboud University Medical Center, Nijmegen, The Netherlands 
9 Imaging Genetics Center, USC Mark and Mary Stevens Neuroimaging and Informatics Institute, Keck School of Medicine of the University of Southern California, Marina Del Rey, CA, USA

10

Department of Human Genetics, Radboud University Medical Center, Nijmegen, The Netherlands

11 Department of Psychiatry, Radboud University Medical Center, Nijmegen, The Netherlands

12 Imaging Genetics Center, Department of Neurology and Biomedical Engineering, USC Mark and Mary Stevens Neuroimaging and Informatics Institute, Keck School of Medicine of USC, University of Southern California, Marina Del Rey, CA, USA

13

Department of Psychiatry, Center for Multimodal Imaging and Genetics (CMIG), University of California, San Diego, CA, USA

14 Centre for Youth Mental Health, The University of Melbourne, Parkville, VIC, Australia

15 Orygen, The National Centre of Excellence for Youth Mental Health, Parkville, VIC, Australia
16 UCL Queen Square Institute of Neurology, Department of Clinical and Experimental Epilepsy, University College London, London, UK

17 Chalfont Centre for Epilepsy, Epilepsy Society, Bucks, UK

18 SA MRC Unit on Risk \& Resilience in Mental Disorders, Department of Psychiatry \& Neuroscience Institute, University of Cape Town, Cape Town, South Africa

19 Department of Psychiatry and Department of Anatomy \& Neurosciences, Amsterdam UMC/VUmc, Amsterdam, The Netherlands

20

Clinical Translational Neuroscience Laboratory, Department of Psychiatry and Human Behavior, University of California Irvine, Irvine, CA, USA

21 Center for the Neurobiology of Learning and Memory, University of California Irvine, Irvine, CA, USA

22 Donders Centre for Cognitive Neuroimaging, Radboud University Medical Center, Nijmegen, The Netherlands

23 Neuro Imaging Institute for Neuroimaging and Informatics, Keck School of Medicine of the University of Southern California, Marina Del Rey, CA, USA 